\title{
Laccase electrochemical biosensor based on graphene-gold/chitosan nanocomposite film for bisphenol a detection
}

\begin{abstract}
Background: Bisphenol A (BPA) is considered one of the most common chemicals that could cause environmental endocrine disrupting. Therefore, there is an increasing demand for simple, rapid and sensitive methods for BPA detection that result from BPA leaching into foods and beverages from storage containers. Herein, a simple laccase electrochemical biosensor was developed for the determination of BPA based on Screen-Printed Carbon Electrode (SPCE) modified graphenegold/ chitosan. The synergic effect of graphenegold/chitosan nanocomposite as electrode modifier greatly facilitates electron-transfer processes between the electrolyte and laccase enzyme, thus leads to a remarkably improved sensitivity for bisphenol A detection. Methods: In this study, laccase enzyme is immobilized onto the Screen-Printed Carbon Electrode (SPCE) modified Graphene-Decorated Gold Nanoparticles (Gr-AuNPs) with Chitosan (Chit). The surface structure of nanocomposite was studied using different techniques including Field Emission Scanning Microscopy (FESEM), TRANSMISSION Electron Microscopy (TEM), Raman spectroscopy and Energy Dispersive $\mathrm{X}$-ray (EDX). Meanwhile, the electrochemical performances of the modified electrodes were studied using Cyclic Voltammetry (CV) and Differential Pulse Voltammetry (DPV). Result: The developed laccase biosensor offered excellent analytical performance for the detection of BPA with a sensitivity of $0.271 \mu \mathrm{A} / \mu \mathrm{M}$ and Limit of Detection (LOD) of $0.023 \mu \mathrm{M}$, respectively. Moreover, the constructed biosensor showed good reproducibility, selectivity and stability towards BPA. The sensor has been used to detect BPA in a different type of commercial plastic products as a real sample and satisfactory result was obtained when compared with the HPLC method. Conclusion: The proposed electrochemical laccase biosensor exhibits good result which is considered as a promising candidate for a simple, rapid and sensitive method especially in the resource- limited condition.
\end{abstract}

Keyword: Bisphenol A; Electrochemical biosensor; Graphene-gold nanocomposite; Laccase; Screen-Printed Carbon Electrode (SPCE); Synergic effect 